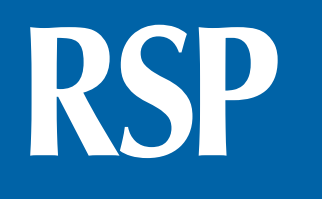

http://www.rsp.fsp.usp.br/
Revista de Saúde Pública

\title{
Neighborhood environmental factors associated with leisure walking in adolescents
}

\author{
Arieli Fernandes Dias' (iD, Anelise Reis Gaya' (iD, Maria Paula Santos" (iD, Caroline Brand' iD, \\ Andreia Nogueira Pizarro" ID, Camila Felin Fochesatto' (iD, Thiago Monteiro Mendes ${ }^{\text {III }}$ (ID, \\ Jorge Mota" iD, Adroaldo Cezar Araujo Gaya' iD \\ ' Universidade Federal do Rio Grande do Sul. Escola de Educação Física, Fisioterapia e Dança. Programa de \\ Pós-Graduação em Ciências do Movimento Humano. Grupo de pesquisa Projeto Esporte Brasil. Porto Alegre, \\ RS, Brasil \\ " Universidade do Porto. Faculdade de Desporto. Centro de Investigação em Atividade Física, Saúde e Lazer. \\ Porto, Portugal \\ III Universidade do Porto. Centro de Estudos em Geografia e Ordenamento do Território. Porto, Portugal
}

Correspondence:

Arieli Fernandes Dias

Rua Felizardo, 750 Jardim Botânico 90690-200 Porto Alegre, RS, Brasil

E-mail: ariieli_dias@hotmail.com

Received: Nov 23, 2019

Approved: Jan 30, 2020

How to cite: Dias AF, Gaya AR, Santos MP, Brand C, Pizarro AN, Fochesatto CF, et al. Neighborhood environmental factors associated with leisure walking in adolescents. Rev Saude Publica. 2020;54:61.

Copyright: This is an open-access article distributed under the terms of the Creative Commons Attribution License, which permits unrestricted use, distribution, and reproduction in any medium, provided that the original author and source are credited.

\section{ABSTRACT}

OBJECTIVE: To verify the associations of leisure walking with perceived and objective measures of neighborhood environmental factors stratified by gender and socioeconomic status (SES) in Brazilian adolescents.

METHODS: Cross-sectional study with a random sample of 1,130 high school students (47.3\% girls; aged 14 to 20 years old) from Porto Alegre, Brazil. Leisure walking and SES were self-reported by the adolescents. Perceived environmental factors were assessed through Neighborhood Environment Walkability Scale for Youth (NEWS-Y). Objective measures were evaluated using Geographic Information Systems, with road network calculated around the adolescent's residential address, using $0.5 \mathrm{~km}$ and $1.0 \mathrm{~km}$ buffers. Data collection was carried out in 2017 and generalized linear regression models were used.

RESULTS: Leisure walking was positively associated with access to services ( $0.5 \mathrm{~km}$ buffers [Odds ratio $(\mathrm{OR})=2.22] 1.0 \mathrm{~km}$ buffers $[\mathrm{OR}=2.17])$ and lower distance to parks and squares $(0.5 \mathrm{~km}[\mathrm{OR}=2.80] 1.0 \mathrm{~km}[\mathrm{OR}=2.73])$ in girls from low SES. Residential density $(0.5 \mathrm{~km}$ $[\mathrm{OR}=1.57] 1.0 \mathrm{~km}[\mathrm{OR}=1.54])$ and walkability index $(0.5 \mathrm{~km}[\mathrm{OR}=1.17] 1.0 \mathrm{~km}[\mathrm{OR}=1.20])$ were associated with leisure walking in girls from middle SES. Boys from low SES showed an inverse association between crime safety and leisure walking $(0.5 \mathrm{~km}$ [OR $=0.59] 1.0 \mathrm{~km}$ [OR $=0.63])$. Neighborhood recreation facilities was positively associated with leisure walking in middle SES $(0.5 \mathrm{~km}[\mathrm{OR}=1.55] 1.0 \mathrm{~km}[\mathrm{OR}=1.60])$. Land use mix $(0.5 \mathrm{~km}$ [OR = 1.81$] 1.0 \mathrm{~km}$ [OR = 1.81]), neighborhood recreation facilities $(0.5 \mathrm{~km}$ [OR $=2.32] 1.0 \mathrm{~km}[\mathrm{OR}=2.28])$ and places for walking $(0.5 \mathrm{~km}[\mathrm{OR}=2.07] 1.0 \mathrm{~km}$ [OR=2.22] $)$ were positively associated with leisure walking in high SES.

CONCLUSION: Environmental factors (objectively and subjectively measured) and leisure walking show association in boys and girls of different SES.

DESCRIPTORS: Adolescent. Walking. Socioeconomic Factors. Built Environment. Environmental Health. Cross-Sectional Studies. 


\section{INTRODUCTION}

All health domains recognize physical activity as beneficial. A study developed in 17 countries, including Brazil, showed that higher physical activity levels lower the risk of mortality and cardiovascular disease in individuals from low, middle, and high-income countries $^{1}$. Additionally, participate in physical activity, especially during leisure time, lowers the risk of incident coronary heart disease among young women ${ }^{2}$. However, the prevalence of leisure-time physical inactivity (lower than $60 \mathrm{~min} /$ day) was $54.3 \%$ in Brazilians adolescents; more than a quarter of adolescents $(26.5 \%)$ reported no engagement in physical activity during leisure time ${ }^{3}$.

A systematic review by Bauman et al. ${ }^{4}$ showed that physical activity in low and middle-income countries is associated with demographic, biological, psychosocial, environmental, social, and cultural variables. In recent years, researchers have shown increased interest in the association between environmental correlates and physical activity. Regarding the young population and the specific domain of leisure physical activity, environmental characteristics such as distance to local facilities and home environment were negatively associated in Portuguese adolescents ${ }^{5}$. In Nigeria, residential density and availability/quality of infrastructures were positively associated with leisure-time moderate to vigorous physical activity (MVPA) in adolescents ${ }^{6}$.

In Brazil, evidences available from Curitiba ${ }^{7,8}$, São Paulo ${ }^{9}$ and Recife ${ }^{10}$ concerned adults, while few studies focused on youngsters. Adolescents from northeastern Brazil reported that "seeing other adolescents engaged in physical activities" and "seeing interesting things while walking" prompted recreational activities ${ }^{11}$. In the south, living near the beach increased leisure-time moderate and vigorous physical activity (MVPA) for adolescents ${ }^{12}$. Lima et al. ${ }^{13}$ identified that distance from home and number of recreational facilities in the neighborhood were associated with physical activity among adolescents, and that these relationships differ according to gender.

Literature about this topic in Brazilian youth is scarce. Therefore, our study adds new information to the field including both objective and perceived measures of the environment for each gender and different socioeconomic status (SES). Also, we explore data from a city without evidence on those aspects. Additionally, in developing countries, information about the built environment by SES can contribute to the development of public policies, increase physical activity and result in a healthier population. Thus, the aim of this study was to verify the associations between leisure walking and perceived and objective measures of neighborhood environmental factors stratified by SES and gender in Brazilian adolescents.

\section{METHODS}

\section{Study Characteristics and Ethical Aspects}

Cross-sectional and quantitative study developed in Porto Alegre, Brazil. The city has a population of approximately 1.4 million inhabitants (year 2010), a territorial area of $496.681 \mathrm{~km}^{2}$ and a demographic density of $2.837,53$ inhabitants $/ \mathrm{km}^{214}$. The study was approved by the Ethics Committee of Research with Human Beings of the Universidade Federal do Rio Grande do Sul (nº. 1,338.597).

\section{Participant Recruitment}

The study sample comprised approximately 34,645 high school students, from 71 public schools ${ }^{15}$, allocated in the following regions: 8,057 north, 6,423 south, 4,268 east, and 15,897 central district.

To calculate the sample size, the following criteria were considered: a) estimated population of 34,645 students $(\mathrm{N})$; b) proportion of $50 \%$ (p); c) complementary percentage 
of 100 - p (q); d) degree of confidence of 2 standard deviations (S); and e) acceptable sampling error of $3 \%$ (e). After adopting these criteria, the formula $\left[n=S^{2}\right.$.p.q.N/e $e^{2}(N-1)$ $+\left(\mathrm{S}^{2}\right.$.p.q)] estimated that 1,077 students should be evaluated, as a representative sample of the population. However, to avoid difficulties with sample loss, an increase of $5 \%$ was assumed, totaling 1,130 adolescents. The power of the test was then calculated in the software $G^{*}$ power version 3.1.9.2, considering generalized linear regression, small effect size $\left(F^{2}=0.03\right), \alpha<0.05$ and 19 predictors. Thus, the power of the test was 0.97 .

Sample selection considered the proportion of youth enrolled in the schools by region; thus comprising: 263 students from 4 schools in the north region (23.26\%); 518 students from 7 schools in the central region (45.88\%); 140 students from 2 schools in the east region (12.32\%); and 209 students from 3 schools in the south region (18.54\%).

Sample selection was performed in multiple phases ${ }^{16}$. First, schools were selected according to each region, and then, in the schools, high school classes were randomly selected. A number was assigned for each school and all numbers were placed in a box, mixed and randomly reelected one by one. Then, data was collected in one class from each high school year. All students from each class selected were invited to participate in the study. Inclusion criteria were: a) belong to the first, second or third year of high school; b) hand in the informed consent form signed by a parent or guardian; and c) sign the assent form. According to Sawyer et al. ${ }^{17}$ 10-24 years range corresponds more closely to adolescent growth and popular understandings of this life phase; thus, we use the term adolescents, even when some students were over 18 years of age.

\section{Data Collection}

Data was collected during an eight-month period in 2017. First, the researcher went to the selected schools, explained the study and if the principals agreed to participate, they were asked to sign an acceptance form. Then data collection was scheduled. Questionnaires were filled out during a regular class, corresponding to approximately 45 minutes and data confidentiality was kept.

\section{Measures}

Leisure physical activity

Self-reported leisure physical activity was assessed by their respective sections of the long form of the International Physical Activity Questionnaire (IPAQ) ${ }^{18}$. This instrument was translated and adapted culturally in several countries ${ }^{18}$, including Brazil, and has been applied in epidemiological studies in Latin American ${ }^{19}$. To assess leisure walking, subjects answered the question 'How many days per week do you usually walk in your free time?'. Response choices ranged from zero to seven. We created one binary outcome: participation in leisure-time walking (or not).

\section{Perceived environment}

To measure perceived neighborhood environmental factors, the version of the Neighborhood Environment Walkability Scale for Youth (NEWS-Y) ${ }^{20}$ validated in Brazil ${ }^{21}$ was used. This questionnaire evaluates perceived environmental factors that may influence youth physical activity $^{20}$. Questions were considered according to the following dimensions, proposed by the NEWS-Y scoring guidelines ${ }^{22}$ : Land use mix-diversity (perception of distance from home to a variety of common destinations, such as shops or school), neighborhood recreation facilities (perceived distance from the student house, walking to a variety of places for physical activity practice, such as walking/running track or large public park), access to services, street connectivity, places for walking, neighborhood aesthetics, neighborhood safety and crime safety. More information can be found in Lima et al..$^{21}$, Rosenberg et al. ${ }^{20}$ and through the link <http://sallis.ucsd.edu/Documents/Measures_documents/NEWS_Y_ adolescent.pdf>. 
For land use mix-diversity and neighborhood recreation facilities, the answer options were: 1-5min, 6-10min, 11-20min, 21-30min, more than $30 \mathrm{~min}$ and don't know/there isn't. The option 'don't know' was coded as "more than 30 min" because if the facility is not within walking distance the actual walk is likely more than 31 minutes $^{22}$. All items were reverse coded and employed mean values.

All the questions from the dimensions access to services, street connectivity, places for walking, neighborhood aesthetics, neighborhood and crime safety were measured using 4-point Likert scale (strongly disagree, partially disagree, partially agree, strongly agree). All determinants were calculated following the NEWS-Y scoring guidelines ${ }^{22}$, with a higher score indicating better conditions for physical activity.

\section{Objective environment factors}

From the adolescent's addresses reported in the questionnaire, we performed a georeferencing process and represented them in the Geographic System Information (GIS) environment through ArcMap 10.3.1 software. The shapefile of the streets, parks and squares provided by the Municipal Department of Urbanism, Environment and Sustainability of Porto Alegre - RS were used for the analyses.

The distances between houses and parks or squares of the city were defined by the tool "Network Analyst/Closest Facility." The distance was calculated in meters and categorized in tertiles of "close," "medium" and "far." Buffers within 0.5km and 1.0km of the participants' homes, reachable by the street network, were defined to estimate accessible neighborhood features. Thus, the following variables were used: existence of parks and squares (existence of parks and squares in buffer); existence of bicycle path (existence of bicycle path in buffer); residential density (number of residences within each buffer); density of blocks (number of blocks within each buffer); average size of the blocks (average size of streets/blocks within each buffer); connectivity between streets (intersection number of streets in buffer); and walkability index (sum $2 *$ z-score connectivity between streets $+\mathrm{z}$-score residential density).

\section{Socioeconomic status}

SES was assessed through a questionnaire that included the number of owned items at the adolescents' residence and the level of schooling of the parent or guardian. By means of the criteria established by the Brazilian Market Research Association ${ }^{23}$, a score was assigned to each answer, and the sum of the points identified each student's economic class ${ }^{23}$. Then adolescents were classified into low $\left(1^{\circ}\right.$ tertile), middle ( $2^{\circ}$ tertile $)$ and high ( $3^{\circ}$ tertile) class.

\section{Data Analyses}

Descriptive data are shown as absolute and relative values, means and standard deviations stratified by gender and SES. Geographical data of the squares, parks, bicycle paths and students' homes are presented in the figure.

The internal consistency from NEWS-Y dimensions variables was verified through Cronbach Alpha (0.89), indicating 0.89 as an acceptable reliability. Values for each dimension were: land use mix-diversity (0.88), neighborhood recreation facilities $(0.84)$, access to services (0.36), street connectivity (0.36), places for walking (0.30), neighborhood aesthetics (0.71), neighborhood safety (0.10) and crime safety $(0.85)$.

Generalized linear regressions were used to test the association between perceived and objective measures of neighborhood environmental factors with leisure walking. Thus, the analyses were splitted by gender and SES into two models: variables of the perceived environment adding objective built environment with 0.5 kilometers buffers (model 1); and perceived environment adding objective built environment with 1.0 kilometer buffers (model 2). All analyses were adjusted for age and region and we tested additional adjustment for the environmental variables considering collinearity between them (rho $\geq 0.60$ ). Variables 



Figure. Georeferencing of students' residence, parks, squares, and bicycle path in Porto Alegre (RS), Brazil, $2017(\mathrm{n}=1010)$.

with high collinearity were described in the legend of the tables. All analyses were carried out using the IBM SPSS 22 (SPSS, Inc., Chicago, Illinois, USA), $\alpha<0.05$ was adopted and confidence intervals (95\%) were presented.

\section{RESULTS}

Although the study sample comprised 1,130 adolescents from Porto Alegre-RS, the SES data refers only to 1,113 students. Sample loss occurred because the adolescents responded the questionnaire inadequately.

Table 1 shows the descriptive characteristics of the sample, as well as data concerning perceived and objective measures of neighborhood environmental factors by sex and SES. We observed a higher prevalence of adolescents that engage in leisure walking in boys and girls from high SES. 
Table 1. Descriptive characteristics of the sample stratified by gender and socioeconomic status in adolescents from Porto Alegre (RS), Brazil, 2017.

\begin{tabular}{|c|c|c|c|c|c|c|}
\hline \multirow{4}{*}{ Variables } & \multicolumn{3}{|c|}{ Girls } & \multicolumn{3}{|c|}{ Boys } \\
\hline & \multicolumn{3}{|c|}{ SES $(n=587)$} & \multicolumn{3}{|c|}{ SES $(n=526)$} \\
\hline & Low $(n=189)$ & Middle $(n=201)$ & High $(n=197)$ & Low $(n=175)$ & Middle $(n=180)$ & High $(n=171)$ \\
\hline & n (\%) & n (\%) & n (\%) & n (\%) & n (\%) & n (\%) \\
\hline \multicolumn{7}{|l|}{ Age } \\
\hline $14-15$ & $32(16.9)$ & $35(17.4)$ & $46(23.4)$ & $23(13.1)$ & 25 (13.9) & $44(25.7)$ \\
\hline $16-17$ & $129(68.3)$ & $136(67.7)$ & $137(69.5)$ & $117(66.9)$ & $118(65.6)$ & $105(61.4)$ \\
\hline $18-20$ & $28(14.8)$ & $30(14.9)$ & $14(7.1)$ & $35(20.0)$ & 37 (20.6) & $22(12.9)$ \\
\hline \multicolumn{7}{|l|}{ Region } \\
\hline Central & 88 (46.6) & $100(49.8)$ & $103(52.3)$ & $58(33.1)$ & $70(38.9)$ & $92(53.8)$ \\
\hline North & $33(17.5)$ & $48(23.9)$ & $48(24.4)$ & $42(24.0)$ & $48(26.7)$ & $28(16.4)$ \\
\hline South & $36(19.0)$ & $33(16.4)$ & $32(16.2)$ & $38(21.7)$ & 39 (21.7) & 39 (22.8) \\
\hline East & 32 (16.9) & $20(10.0)$ & $14(7.1)$ & $37(21.1)$ & $23(12.8)$ & $12(7.0)$ \\
\hline \multicolumn{7}{|l|}{ Leisure PA } \\
\hline Leisure Walking (proportion: yes) & $97(51.3)$ & $94(46.8)$ & $107(54.3)$ & $103(58.9)$ & $102(56.7)$ & $111(64.9)$ \\
\hline \multicolumn{7}{|l|}{ Objective (GIS) } \\
\hline Existence of parks and squares $(0.5 \mathrm{~km})$ & $81(48.2)$ & 109 (59.9) & $109(61.6)$ & $98(62.0)$ & $103(63.6)$ & $103(67.8)$ \\
\hline Existence of parks and squares $(1.0 \mathrm{~km})$ & $132(78.6)$ & $160(87.9)$ & $156(88.1)$ & $134(84.8)$ & $136(84.0)$ & $141(92.8)$ \\
\hline Existence of bicycle path $(0.5 \mathrm{~km})$ & $12(7.1)$ & $16(8.8)$ & $27(15.3)$ & $26(16.5)$ & $24(14.8)$ & $15(9.9)$ \\
\hline \multirow[t]{2}{*}{ Existence of bicycle path $(1.0 \mathrm{~km})$} & $30(17.9)$ & $44(24.2)$ & $50(28.2)$ & $43(27.2)$ & $37(22.8)$ & $35(23.0)$ \\
\hline & Mean (SD) & Mean (SD) & Mean (SD) & Mean (SD) & Mean (SD) & Mean (SD) \\
\hline \multicolumn{7}{|l|}{ Perceived Environment } \\
\hline Land use mix - diversity & $3.16(0.85)$ & $3.54(0.89)$ & $3.73(0.84)$ & $3.45(0.81)$ & $3.66(0.81)$ & $3.83(0.94)$ \\
\hline Neighborhood recreation facilities & $2.25(0.87)$ & $2.73(1.05)$ & $2.97(0.96)$ & $2.82(0.99)$ & $3.03(1.04)$ & $3.49(1.00)$ \\
\hline Access to services & $2.81(0.51)$ & $2.97(0.51)$ & $3.01(0.48)$ & $2.98(0.48)$ & $2.93(0.47)$ & $3.05(0.45)$ \\
\hline Street connectivity & $2.68(0.68)$ & $2.69(0.70)$ & $2.79(0.67)$ & $2.75(0.68)$ & $2.76(0.67)$ & $2.81(0.66)$ \\
\hline Places for walking & $2.54(0.80)$ & $2.79(0.69)$ & $2.77(0.66$ & $2.81(0.74)$ & $2.75(0.59)$ & $2.87(0.61)$ \\
\hline Neighborhood aesthetics & $2.30(0.73)$ & $2.50(0.69)$ & $2.70(0.67)$ & $2.55(0.73)$ & $2.56(0.71)$ & $2.76(0.69)$ \\
\hline Neighborhood safety & $2.56(0.48)$ & $2.44(0.44)$ & $2.37(0.47)$ & $2.28(0.42)$ & $2.32(0.41)$ & $2.29(0.47)$ \\
\hline Crime safety & $2.86(0.82)$ & $2.76(0.80)$ & $2.59(0.76)$ & $2.38(0.79)$ & $2.46(0.73)$ & $2.36(0.78)$ \\
\hline \multicolumn{7}{|l|}{ Objective (GIS) } \\
\hline Lower distance for parks and squares & $\begin{array}{c}818.90 \\
(1,089.89)\end{array}$ & $558.43(611.24)$ & $611.98(999.72)$ & $\begin{array}{c}649.18 \\
(1,055.49)\end{array}$ & $\begin{array}{c}696.78 \\
(1,246.78)\end{array}$ & $\begin{array}{c}453.92 \\
(457.31)\end{array}$ \\
\hline Lower distance for bicycle path & $\begin{array}{c}3,026.72 \\
(2,094.62)\end{array}$ & $\begin{array}{c}2,507.85 \\
(1,757.05)\end{array}$ & $\begin{array}{c}2,285.94 \\
(1,913.92)\end{array}$ & $\begin{array}{c}2,607.55 \\
(1,986.55)\end{array}$ & $\begin{array}{c}2,628.14 \\
(2,015.07)\end{array}$ & $\begin{array}{c}2,359.63 \\
(1,621.13)\end{array}$ \\
\hline \multicolumn{7}{|c|}{ Objective Environment Factors (0.5 km-buffers) } \\
\hline Residential density & $\begin{array}{c}2,550.35 \\
(1,254.49)\end{array}$ & $\begin{array}{c}2,855.59 \\
(1,456.52)\end{array}$ & $\begin{array}{c}2,811.36 \\
(1,540.46)\end{array}$ & $\begin{array}{c}2,728.11 \\
(1,461.43)\end{array}$ & $\begin{array}{c}2,583.06 \\
(1,373.02)\end{array}$ & $\begin{array}{c}3,003.98 \\
(1,578.16)\end{array}$ \\
\hline Connectivity between streets & $53.61(33.53)$ & $51.94(31.46)$ & $47.20(27.46)$ & $50.83(33.42)$ & $49.84(33.10)$ & $50.11(27.92)$ \\
\hline Blocks density & $\begin{array}{c}7,973.63 \\
(3,469.49)\end{array}$ & $\begin{array}{c}8,765.06 \\
(3,516.94)\end{array}$ & $\begin{array}{c}8,780.96 \\
(3,409.79)\end{array}$ & $\begin{array}{c}8,627.06 \\
(3,893.09)\end{array}$ & $\begin{array}{c}8,515.05 \\
(3,774.66)\end{array}$ & $\begin{array}{c}9,231.46 \\
(3,279.48)\end{array}$ \\
\hline Average size of the blocks & $\begin{array}{c}120.15 \\
(123.73)\end{array}$ & $121.61(63.89)$ & $134.17(123.79)$ & $118.99(54.31)$ & $118.76(44.33)$ & $122.75(44.81)$ \\
\hline Walkability index* & $0.02(2.69)$ & $0.15(2.54)$ & $-0.19(2.25)$ & $-0.05(2.64)$ & $-0.17(2.63)$ & $0.15(2.29)$ \\
\hline \multicolumn{7}{|c|}{ Objective Environment Factors (1.0 km-buffers) } \\
\hline Residential density & $\begin{array}{c}6,179.16 \\
(3,488.29)\end{array}$ & $\begin{array}{c}7,464.60 \\
(4,053.18)\end{array}$ & $\begin{array}{c}7,862.40 \\
(4,562.06)\end{array}$ & $\begin{array}{c}6,989.69 \\
(4,136.42)\end{array}$ & $\begin{array}{c}6,970.60 \\
(3,727.70)\end{array}$ & $\begin{array}{c}8,086.56 \\
(4,368.71)\end{array}$ \\
\hline Connectivity between streets & $\begin{array}{c}180.61 \\
(104.48)\end{array}$ & $193.36(104.54)$ & $178.90(94.90)$ & $\begin{array}{c}185.15 \\
(107.83)\end{array}$ & $187.33(111.07)$ & $186.56(92.36)$ \\
\hline Blocks density & $\begin{array}{c}25,397.55 \\
(11,714.66)\end{array}$ & $\begin{array}{c}29,766.52 \\
(11,850.19)\end{array}$ & $\begin{array}{c}30,480.68 \\
(12,090.82)\end{array}$ & $\begin{array}{c}28,926.19 \\
(13,294.79)\end{array}$ & $\begin{array}{c}29,464.51 \\
(12,476.15)\end{array}$ & $\begin{array}{c}30,918.12 \\
(10,734.35)\end{array}$ \\
\hline Average size of the blocks & $107.47(40.74)$ & $112.55(44.83)$ & $121.65(43.56)$ & $112.99(39.70)$ & $116.68(39.55)$ & $118.53(39.69)$ \\
\hline Walkability index* & $-0.35(2.62)$ & $0.20(2.58)$ & $-0.01(2.45)$ & $-0.06(2.68)$ & $-0.07(2.69)$ & $0.22(2.31)$ \\
\hline
\end{tabular}

*Standardized variables (transformed into z-scores). 
Table 2. Association between perceived and objective neighborhood environmental factors and leisure walking by socioeconomic status in adolescent girls from Porto Alegre (RS), Brazil, 2017.

\begin{tabular}{|c|c|c|c|c|c|c|}
\hline \multirow{5}{*}{ Perceived Environment } & \multicolumn{6}{|c|}{ Leisure walking } \\
\hline & \multicolumn{3}{|c|}{ Model $1^{\text {a }}$} & \multicolumn{3}{|c|}{ Model $2^{\text {a }}$} \\
\hline & \multicolumn{3}{|c|}{ SES $(n=587)$} & \multicolumn{3}{|c|}{ SES $(n=587)$} \\
\hline & Low $(n=189)$ & Middle $(n=201)$ & High $(n=197)$ & Low $(n=189)$ & Middle $(n=201)$ & High $(n=197)$ \\
\hline & OR $(\mathrm{Cl}-95 \%)$ & OR $(\mathrm{Cl}-95 \%)$ & OR (Cl-95\%) & OR (Cl-95\%) & OR (Cl-95\%) & OR (Cl-95\%) \\
\hline Land use mix - diversity & $0.79(0.48-1.33)$ & $1.20(0.81-1.76)^{b}$ & $1.18(0.73-1.90)$ & $0.79(0.47-1.32)$ & $1.07(0.70-1.63)^{b}$ & $1.17(0.71-1.93)$ \\
\hline Neighborhood recreation facilities & $0.76(0.46-1.25)$ & $1.33(0.95-1.86)^{b}$ & $1.19(0.79-1.79)$ & $0.81(0.50-1.32)$ & $1.14(0.81-1.60)^{b}$ & $1.09(0.73-1.62)$ \\
\hline Access to services & $2.22(1.01-4.92)$ & $1.12(0.58-2.16)$ & $0.92(0.42-2.02)$ & $2.17(1.01-4.83)$ & $0.99(0.50-1.97)$ & $0.93(0.42-2.05)$ \\
\hline Street connectivity & $0.72(0.43-1.21)$ & $1.13(0.68-1.87)$ & $1.27(0.74-2.16)$ & $0.69(0.40-1.17)$ & $1.02(0.62-1.67)$ & $1.41(0.83-2.40)$ \\
\hline Places for walking & $0.74(0.46-1.18)$ & $0.80(0.45-1.42)$ & $1.03(0.60-1.74)$ & $0.80(0.50-1.26)$ & $0.87(0.50-1.53)$ & $1.01(0.59-1.71)$ \\
\hline Neighborhood aesthetics & $1.09(0.65-1.81)$ & $1.30(0.78-2.17)$ & $1.51(0.87-2.63)$ & $1.12(0.68-1.85)$ & $1.20(0.73-1.98)$ & $1.38(0.80-2.39)$ \\
\hline Neighborhood safety & $0.81(0.35-1.84)$ & $1.14(0.53-2.45)$ & $0.65(0.29-1.46)$ & $0.89(0.39-1.99)$ & $1.19(0.56-2.55)$ & $0.68(0.30-1.54)$ \\
\hline Crime safety & $0.93(0.61-1.41)$ & $0.83(0.54-1.28)$ & $0.75(0.47-1.20)$ & $0.91(0.59-1.38)$ & $0.82(0.54-1.27)$ & $0.71(0.44-1.15)$ \\
\hline
\end{tabular}

\section{Objective (GIS)}

Lower distance for parks and squares

$$
\begin{aligned}
& 1 \text { tertile (close) } \\
& 2 \text { tertile (medium) } \\
& 3 \text { tertile (far) }
\end{aligned}
$$

Lower distance for bicycle path

$$
\begin{aligned}
& 1 \text { tertile (close) } \\
& 2 \text { tertile (medium) } \\
& 3 \text { tertile (far) }
\end{aligned}
$$

\section{Objective Environment Factors}

Existence of parks and squares

$$
\begin{aligned}
& \text { No } \\
& \text { Yes }
\end{aligned}
$$

1

$2.80(1.04-7.55)^{b}$

$2.35(0.93-5.94)^{\mathrm{b}}$

$0.56(0.22-1.39)^{b}$

$0.42(0.15-1.16)^{b}$

$2.73(1.01-7.32)$

$0.47(0.18-1.19)$

$0.55(0.20-1.48)$

1

$1.88(0.72-4.88)$

$2.09(0.86-5.04)$

$0.51(0.19-1.32)^{b}$

$1.91(0.68-5.36)^{b}$

$2.14(0.86-5.31)^{b}$

$0.57(0.21-1.50)^{b}$

1.37 (0.63-2.95)

1

$1.93(0.85-4.35)$

$0.48(0.20-1.17)^{b}$

$1.42(0.58-3.42)^{\mathrm{b}}$

1

(0.5km - buffers)

1

$1.75(0.78-3.96)^{b}$

$0.52(0.21-1.30)^{\mathrm{b}}$

1

(1 km - buffers)

1

$1.63(0.75-3.53)^{b} \quad 0.74(0.36-1.52)^{b}$

1

$0.73(0.34-1.58)^{b}$

$1.04(0.45-2.43)^{b}$

$1.54(0.49-4.80)$

$0.30(0.07-1.17)$

Existence of bicycle path

No

Yes

Residential density*

Connectivity between streets*

Blocks density*

Average size of the blocks*

Walkability index*
$2.16(0.47-9.93)$

$0.99(0.62-1.57)^{\mathrm{b}}$

$0.92(0.65-1.30)^{b}$

$1.11(0.77-1.61)^{\mathrm{b}}$

$1.08(0.83-1.42)^{\mathrm{b}}$

$0.93(0.81-1.08)^{\mathrm{b}}$
1

$1.80(0.52-6.16)$

$1.57(1.08-2.28)^{b}$

$1.25(0.89-1.76)^{\mathrm{b}}$

$1.16(0.79-1.71)^{\mathrm{b}}$

$0.90(0.57-1.43)^{b}$

$1.17(1.02-1.35)^{b}$
1

$0.77(0.31-1.91)^{b} \quad 1.70(0.68-4.26)^{b}$

$1.21(0.83-1.75)^{\mathrm{b}} \quad 0.88(0.51-1.51)^{\mathrm{b}}$

$1.01(0.67-1.51)^{\mathrm{b}} \quad 1.02(0.72-1.43)^{\mathrm{b}}$

$1.11(0.74-1.58)^{\mathrm{b}}$

$0.86(0.58-1.29)^{\mathrm{b}}$

$1.00(0.68-1.47)^{\mathrm{b}}$

$1.12(0.76-1.67)^{\mathrm{b}}$

$0.94(0.80-1.09)^{\mathrm{b}}$ $0.99(0.44-2.20)^{b} \quad 0.79(0.37-1.69)^{b}$

$1.54(\mathbf{1 . 0 5}-\mathbf{2 . 2 5})^{\mathrm{b}} \quad 1.25(0.84-1.84)^{\mathrm{b}}$

$1.14(0.80-1.61)^{\mathrm{b}} \quad 1.90(0.64-1.45)^{\mathrm{b}}$

$1.35(0.92-1.98)^{\mathrm{b}} \quad 1.13(0.72-1.77)^{\mathrm{b}}$

$0.78(0.51-1.19)^{\mathrm{b}} \quad 0.77(0.52-1.15)^{\mathrm{b}}$

$1.20(\mathbf{1 . 0 4}-\mathbf{1 . 3 9})^{\mathrm{b}} \quad 1.06(0.89-1.26)^{\mathrm{b}}$

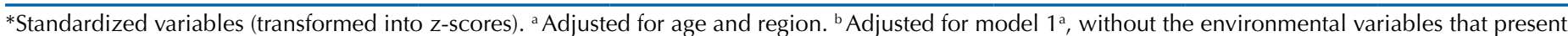
high collinearity ( $r$ ho $\geq 0.60$ ).

Low $(0.5 \mathrm{~km}$ buffers) - High correlation between following variables: Residential density with blocks density (rho $=0.72)$, with connectivity between streets (rho $=0.66)$ and walkability $($ rho $=0.81$ ); Blocks density with connectivity between streets $($ rho $=0.86)$, lower distance for park and squares (rho $=-0.61)$, existence of park and square $(r h o=0.60)$ and walkability $(r h o=0.89)$. Connectivity between streets with average size of the blocks $($ rho $=-0.80)$ and walkability $($ rho $=0.95)$. Average size of the blocks with walkability (rho $=-0.73$ ). Existence of park and square with lower distance for park and square $($ rho $=-0.84)$.

Low ( $1 \mathrm{~km}$ buffers) - High correlation between following variables: Residential density with connectivity between streets (rho $=0.77)$, blocks density $($ rho $=0.85)$, existence of park and square $(r h o=0.60)$ and walkability $(r h o=0.87)$. Connectivity between streets with blocks density $(r h o=0.90)$, average size of the blocks $($ rho $=-0.69)$, existence of park and square $($ rho $=0.61)$ and walkability $($ rho $=0.96)$. Blocks density with walkability $($ rho $=0.93)$ and existence of park and square $(r h o=0.63)$. Walkability with average size of the blocks $($ rho $=-0.61)$ and existence of park and square $($ rho $=0.62)$. Lower distance for bicycle path with existence of bicycle path (rho $=-0.64)$.

Middle ( $0.5 \mathrm{~km}$ buffers) - High correlation between following variables: Land use mix with neighborhood recreation facilities (rho $=0.61)$; residential density with blocks density $(\mathrm{rho}=0.62)$ and walkability $($ rho $=0.76)$. Blocks density with connectivity between streets $($ rho $=0.75)$ and walkability $($ rho $=0.82)$; connectivity between streets with average size of the blocks (rho $=-0.79)$ and walkability $($ rho $=0.87)$. Average size of the blocks with walkability $($ rho $=-0.62)$. Existence of park and square with lower distance for park and square $($ rho $=-0.82)$.

Middle ( $1 \mathrm{~km}$ buffers $)$ - High correlation between following variables: Land use mix with neighborhood recreation facilities $($ rho $=0.61)$. Residential density with connectivity between streets $(\mathrm{rho}=0.76)$ and walkability ( $\mathrm{rho}=0.78$ ). Connectivity between streets with blocks density $($ rho $=0.81)$, average size of the blocks $($ rho $=-0.72)$ and walkability $(r h o=0.93)$. Blocks density with walkability $(r h o=0.89)$. Existence of bicycle path with lower distance for bicycle path $($ rho $=-0.70)$. High $(0.5 \mathrm{~km}$ buffers $)$ - High correlation between following variables: Residential density with blocks density $($ rho $=0.66)$ and walkability $(\mathrm{rho}=0.76)$; Blocks density with connectivity between streets $($ rho $=0.72)$ and walkability ( $r$ ho $=0.82)$; connectivity between streets with average size of the blocks $($ rho $=-0.75)$ and walkability $($ rho $=0.85)$. Existence of park and square with lower distance for park and square $($ rho $=-0.79)$. Existence of bicycle path with lower distance for bicycle path (rho $=-0.62$ ).

High ( $1 \mathrm{~km}$ buffers) - High correlation between following variables: Residential density with blocks density $(\mathrm{rho}=0.75)$ and walkability $(\mathrm{rho}=0.80)$. Connectivity between streets with blocks density $(\mathrm{rho}=0.78)$, average size of the blocks $($ rho $=-0.69)$ and walkability $($ rho $=0.86)$. Blocks density with walkability $($ rho $=0.87)$. Existence of bicycle path with lower distance for bicycle path (rho $=-0.68)$. 
Table 3. Association between perceived and objective neighborhood environment factors and leisure walking by socioeconomic status in adolescent boys from Porto Alegre (RS), Brazil, 2017.

\begin{tabular}{|c|c|c|c|c|c|c|}
\hline \multirow{5}{*}{ Perceived Environment } & \multicolumn{6}{|c|}{ Leisure walking } \\
\hline & \multicolumn{3}{|c|}{ Model $1^{a}$} & \multicolumn{3}{|c|}{ Model $2^{\mathrm{a}}$} \\
\hline & \multicolumn{3}{|c|}{ SES $(n=526)$} & \multicolumn{3}{|c|}{ SES $(n=526)$} \\
\hline & Low $(n=175)$ & Middle $(n=180)$ & High $(n=171)$ & Low $(n=175)$ & Middle $(n=180)$ & High $(n=171)$ \\
\hline & OR (Cl-95\%) & OR $(\mathrm{Cl}-95 \%)$ & OR $(\mathrm{Cl}-95 \%)$ & OR (Cl-95\%) & OR (Cl-95\%) & OR (Cl-95\%) \\
\hline Land use mix - diversity & $0.84(0.47-1.51)$ & $1.30(0.81-2.07)^{b}$ & $1.81(1.09-3.02)^{b}$ & $0.84(0.47-1.51)$ & $1.40(0.91-2.18)^{b}$ & $1.81(1.11-2.94)^{b}$ \\
\hline Neighborhood recreation facilities & $1.45(0.89-2.36)$ & $1.55(1.06-2.29)^{b}$ & $2.32(1.26-3.93)^{b}$ & $1.38(0.85-2.23)$ & $1.60(1.09-2.35)^{b}$ & $2.28(1.35-3.86)^{b}$ \\
\hline Access to services & $0.59(0.24-1.45)$ & $1.40(0.64-3.02)$ & $1.06(0.75-1.51)$ & $0.57(0.23-1.40)$ & $1.27(0.57-2.79)$ & $1.09(0.77-1.55)$ \\
\hline Street connectivity & $1.01(0.60-1.71)$ & $0.84(0.50-1.43)$ & $0.57(0.30-1.08)$ & $1.04(0.61-1.78)$ & $0.94(0.55-1.63)$ & $0.56(0.30-1.04)$ \\
\hline Places for walking & $0.94(0.56-1.57)$ & $1.07(0.57-2.00)$ & $2.07(1.03-4.19)$ & $1.01(0.60-1.69)$ & $0.99(0.53-1.86)$ & $2.22(1.10-4.46)$ \\
\hline Neighborhood aesthetics & $1.15(0.68-1.96)$ & $1.01(0.60-1.70)$ & $1.54(0.85-2.78)$ & $1.05(0.62-1.77)$ & $0.89(0.52-1.53)$ & $1.47(0.82-2.63)$ \\
\hline Neighborhood safety & $1.59(0.58-4.38)$ & $1.31(0.56-3.06)$ & $1.15(0.46-2.90)$ & $1.44(0.53-3.91)$ & $1.36(0.58-3.17)$ & $1.34(0.53-3.38)$ \\
\hline Crime safety & $0.59(0.37-0.95)$ & $0.92(0.58-1.48)$ & $0.93(0.55-1.56)$ & $0.63(0.36-0.98)$ & $0.89(0.55-1.45)$ & $0.87(0.53-1.42)$ \\
\hline \multicolumn{7}{|l|}{ Objective (GIS) } \\
\hline \multicolumn{7}{|l|}{ Lower distance for parks and squares } \\
\hline 1 tertile (close) & $0.42(0.15-1.14)^{b}$ & $0.33(0.11-1.01)^{b}$ & $0.40(0.15-1.04)^{b}$ & $0.37(0.13-1.01)$ & $0.35(0.11-1.02)$ & $0.51(0.18-1.46)$ \\
\hline 2 tertile (medium) & $0.47(0.18-1.23)^{\mathrm{b}}$ & $0.67(0.27-1.66)^{b}$ & $0.74(0.26-2.09)^{b}$ & $0.40(0.14-1.11)$ & $0.67(0.23-1.67)$ & $0.91(0.31-2.66)$ \\
\hline 3 tertile (far) & 1 & 1 & 1 & 1 & 1 & 1 \\
\hline \multicolumn{7}{|l|}{ Lower distance for bicycle path } \\
\hline 1 tertile (close) & $0.86(0.33-2.24)$ & $1.13(0.36-3.51)$ & $0.51(0.14-1.80)$ & $0.89(0.33-2.41)^{b}$ & $1.61(0.53-4.87)^{b}$ & $0.50(0.15-1.66)^{b}$ \\
\hline 2 tertile (medium) & $1.93(0.75-4.98)$ & $0.78(0.32-1.87)$ & $0.35(0.10-1.21)$ & $1.83(0.71-4.74)^{b}$ & $0.77(0.30-1.93)^{b}$ & $0.43(0.12-1.48)^{b}$ \\
\hline 3 tertile (far) & 1 & 1 & 1 & 1 & 1 & 1 \\
\hline Objective Environment Factors & & (0.5km - buffers) & & & (1 km - buffers) & \\
\hline \multicolumn{7}{|l|}{ Existence of parks and squares } \\
\hline No & 1 & 1 & 1 & 1 & 1 & 1 \\
\hline Yes & $1.46(0.60-3.53)^{b}$ & $0.80(0.36-1.77)^{b}$ & $0.49(0.20-1.17)^{b}$ & $2.77(0.74-10.30)$ & $0.91(0.28-2.97)$ & $0.96(0.20-4.50)$ \\
\hline \multicolumn{7}{|l|}{ Existence of bicycle path } \\
\hline No & 1 & 1 & 1 & 1 & 1 & 1 \\
\hline Yes & $0.79(0.57-1.09)$ & $2.70(0.93-7.85)$ & $0.35(0.10-1.22)$ & $2.30(0.98-5.25)^{b}$ & $1.73(0.71-4.24)^{b}$ & $0.43(0.15-1.18)^{b}$ \\
\hline Residential density* & $1.21(0.79-1.85)^{\mathrm{b}}$ & $0.80(0.55-1.17)^{b}$ & $1.07(0.70-1.64)^{b}$ & $1.31(0.86-1.99)^{\mathrm{b}}$ & $0.70(0.43-1.16)^{b}$ & $1.16(0.74-1.83)^{b}$ \\
\hline Connectivity between streets* & $1.27(0.84-1.94)^{b}$ & $1.05(0.73-1.50)^{b}$ & $1.03(0.61-1.74)^{b}$ & $1.01(0.66-1.56)^{b}$ & $1.10(0.75-1.61)^{b}$ & $0.61(0.36-1.04)^{b}$ \\
\hline Blocks density* & $1.56(0.96-2.53)^{b}$ & $0.69(0.47-1.02)^{b}$ & $1.31(0.77-2.25)^{b}$ & $1.37(0.86-2.18)^{b}$ & $0.76(0.49-1.16)^{b}$ & $0.97(0.56-1.68)^{b}$ \\
\hline Average size of the blocks* & $0.90(0.50-1.61)^{b}$ & $1.12(0.56-2.25)^{b}$ & $1.23(0.56-2.69)^{b}$ & $1.21(0.75-1.98)^{b}$ & $0.96(0.64-1.45)^{b}$ & $1.46(0.87-2.43)^{b}$ \\
\hline Walkability index* & $1.09(0.93-1.28)^{\mathrm{b}}$ & $1.02(0.88-1.17)^{\mathrm{b}}$ & $0.97(0.81-1.17)^{b}$ & $1.13(0.96-1.33)^{b}$ & $1.01(0.88-1.17)^{\mathrm{b}}$ & $0.87(0.72-1.04)^{b}$ \\
\hline
\end{tabular}

*Standardized variables (transformed into $z$-scores). ${ }^{\mathrm{a}}$ Adjusted for age and region. ${ }^{\mathrm{b}}$ Adjusted for model $1^{\mathrm{a}}$, without the environmental variables that present high collinearity ( $r$ ho $\geq 0.60)$.

Low (0.5km buffers) - High correlation between following variables: Residential density with blocks density $($ rho $=0.66)$ and walkability $($ rho $=0.79)$; Blocks density with connectivity between streets $($ rho $=0.84)$ and walkability $($ rho $=0.89)$. Connectivity between streets with average size of the blocks $($ rho $=-0.69)$ and walkability $($ rho $=0.90)$; Existence of park and square with lower distance for park and square $($ rho $=-0.86)$.

Low ( $1 \mathrm{~km}$ buffers) - High correlation between following variables: Residential density with connectivity between streets (rho $=0.63$ ), blocks density $($ rho $=0.77)$ and walkability $($ rho $=0.76)$. Connectivity between streets with blocks density $($ rho $=0.87)$, average size of the blocks $($ rho $=-0.65)$ and walkability $($ rho $=0.91)$. Blocks density with walkability $(\mathrm{rho}=0.88)$. Lower distance for bicycle path with existence of bicycle path $($ rho $=-0.74)$. Middle (0.5 km buffers) - High correlation between following variables: Land use mix with neighborhood recreation facilities (rho $=0.66)$; residential density with blocks density $(\mathrm{rho}=0.69)$ and walkability $(\mathrm{rho}=0.78)$. Blocks density with connectivity between streets $(\mathrm{rho}=0.78)$ and walkability $($ rho $=0.82)$; connectivity between streets with average size of the blocks $(r h o=-0.75)$ and walkability $($ rho $=0.88)$. Average size of the blocks with walkability $($ rho $=-0.60)$. lower distance for park and square with existence of park and square $($ rho $=-0.84)$ and blocks density $($ rho $=0.62)$. Middle ( $1 \mathrm{~km}$ buffers) - High correlation between following variables: Land use mix with neighborhood recreation facilities (rho $=0.66)$. Residential density with connectivity between streets $(\mathrm{rho}=0.65)$, blocks density $(\mathrm{rho}=0.80)$ and walkability $(\mathrm{rho}=0.81)$. Connectivity between streets with blocks density $($ rho $=0.86)$, average size of the blocks $($ rho $=-0.76)$ and walkability $($ rho $=0.90)$. Walkability with blocks density $($ rho $=0.90)$ and average size of the blocks (rho $=-0.60)$. Existence of bicycle path with lower distance for bicycle path (rho $=-0.68$ ).

High $(0.5 \mathrm{~km}$ buffers $)$ - High correlation between following variables: Land use mix with neighborhood recreation facilities $(\mathrm{rho}=0.68)$. Residential density with blocks density $(\mathrm{rho}=0.67)$ and walkability $(\mathrm{rho}=0.78)$. Blocks density with connectivity between streets $($ rho $=0.70)$ and walkability $($ rho $=0.77)$. Connectivity between streets with average size of the blocks $($ rho $=-0.76)$ and walkability $($ rho $=0.81)$. Existence of park and square with lower distance for park and square (rho $=-0.82)$.

High ( $1 \mathrm{~km}$ buffers) - High correlation between following variables: Land use mix with neighborhood recreation facilities $($ rho $=0.68)$; Residential density with blocks density $($ rho $=0.74)$ and walkability $($ rho $=0.73)$. Connectivity between streets with blocks density $($ rho $=0.73)$, average size of the blocks $($ rho $=-0.75)$ and walkability $($ rho $=0.83)$. Blocks density with walkability $(r h o=0.81)$. Existence of bicycle path with lower distance for bicycle path $($ rho $=-0.64)$. 
The geocoding of residential addresses for built environmental analysis (objective measures) comprised 1,010 adolescents. Sample losses correspond to lack of address information and incompatibilities in the street network. The Figure presents the environmental characteristics, such as existence of bicycle path, parks, and squares, as well as georeferencing of students' residence. We observe an unequal distribution of park and squares around the city, and few bicycle paths.

Table 2 and 3 show the results of perceived and observed neighborhood environmental factors and leisure walking by gender and SES. Girls in 0.5 and 1.0km buffers had similar results (Table 2. Model 1 and 2). The analysis for low SES adolescent girls indicated that access to services and lower distance for parks and squares were positively associated with walking leisure. The objective measures, such as residential density and walkability index, were associated with walking leisure in middle SES. Girls of high SES showed no associations.

Regarding boys, only perceived environmental factors were associated with leisure walking in both models (Table 3). For boys from low SES showed an inverse association between crime safety and leisure walking. Neighborhood recreation facilities was positively associated with leisure walking in middle SES. Land use mix, neighborhood recreation facilities and places for walking were positively associated with leisure walking in high SES.

\section{DISCUSSION}

The main findings of this study show an association between leisure walking and perceived and objective measures of neighbourhood environmental factors, regarding gender and SES in adolescents.

For girls, results indicated that access to services and lower distance to parks and squares were positively associated with leisure walking in low SES. Studies in Argentina ${ }^{24}$ and Brazil ${ }^{13}$ present similar results, adjusting for SES. Additionally, Pereira et al. ${ }^{5}$ analyses disregarded SES, which may have influenced the results as evidence shows that SES affect the relationship between environmental factors and leisure physical activity.

Our study also showed that residential density and walkability index are associated with leisure walking in middle SES girls. Some studies have indicated association between walkability and physical activity in adolescents, but in low and high SES ${ }^{25,26}$. One explanation for this discrepancy can be that these studies considered neighbourhood SES levels instead of individual ones. Additionally, residential density was positively related to walking in adolescents when adjusted for SES.

Results of this study expand on the current knowledge about associations of leisure walking with both perceived and objective measures of neighbourhood environment in adolescents, when considering the impact SES and gender have in leisure physical activity. Most of the evidence on this topic originated from developed countries, where SES may represent a non-intervenient factor.

The lack of association between environmental factors and leisure walking in high SES was unexpected. With no apparent reason for this outcome, we hypothesize that variables unaddressed in this study, such as social support or owning a car, may be influencing this result. Furthermore, adolescents of high SES may have more access to training courses and gyms, which could decrease the time availability for leisure walking. Among girls, a shorter distance to squares and parks, a favourable perception of access in the neighborhood as well as high walkability index and residential density are intervenient factors for leisure walking, and these results varied according to SES.

Among boys, crime safety was inversely associated with leisure walking in low SES. We expected to find similar association in middle and high SES, considering the high crime rates in Brazil and that perceived lack of safety constrains physical activity behaviours ${ }^{27}$. 
Agreeing with our result, Mitáš et al. ${ }^{28}$ indicated that boys who met recommendations for leisure-time walking were the ones who perceived the safest neighborhood environment. In middle and high SES, our results showed that neighborhood recreation facilities were associated with leisure walking, besides land use mix and places for walking. Even though some studies point to the association between shorter distance to parks and number of facilities near home with physical activity in boys ${ }^{13,29}$, literature lacks evidence regarding the influence of SES in this relationship Additionally, systematic reviews have shown that access and neighborhood park environment characteristics was associated with physical activity $^{30}$. Therefore, data concerning different SES indicated that perceived environmental factors, such as crime safety, land use mix, neighborhood recreation facilities and places for walking are important factors for leisure walking in adolescent boys.

We encountered some limitations when interpreting results. The cross-sectional design does not allow for cause and effect to be determined. Additionally, some characteristics that can be associated with the outcome were not assessed, such as motivation and social support. The walkability index did not consider land use mix and retail floor area ratio. Finally, leisure walking was self-reported.

Strengths of this study are the large sample size and using both perceived and objective measures of the environmental factors. Additionally, to our knowledge this was one of the first studies to account for SES and gender in the association between environmental factors and leisure walking in Brazilian adolescents. Considering these aspects, our findings can help public administrations develop strategies to promote physical activity, since some environment characteristics may be important tools to increase healthy behaviour in the population. Also, our results serve to understand how these relationships take place in each gender and SES, thereby contributing to the development of targeted actions for each context. Future studies should use longitudinal designs to analyse the association between environmental factors and leisure walking over time. Another issue is to understand the reasons and other social factors that lead adolescents to leisure walking.

In conclusion, we found an association between environmental factors (objectively and subjectively measured) and leisure walking in boys and girls of different SES. The influence of gender and SES must be considered when approaching environment and physical activity in Brazilian adolescents.

\section{REFERENCES}

1. Lear SA, Hu W, Rangarajan S, Gasevic D, Leong D, Iqbal R, et al. The effect of physical activity on mortality and cardiovascular disease in 130000 people from 17 high-income, middle-income, and low-income countries: the PURE study. Lancet. 2017;390(10113):2643-54. https://doi.org/10.1016/S0140-6736(17)31634-3

2. Chomistek AK, Henschel B, Eliassen AH, Mukamal KJ, Rimm EB. Frequency, type, and volume of leisure-time physical activity and risk of coronary heart disease in young women. Circulation. 2017;134(4):290-9. https://doi.org/10.1161/CIRCULATIONAHA.116.021516

3. Cureau FV, Silva TLN, Bloch KV, Fujimori E, Belfort DR, Carvalho KMB, et al. ERICA: Leisuretime physical inactivity in Brazilian adolescents. Rev Saude Publica. 2016;50 Suppl 1:1s-11s. https://doi.org/10.1590/s01518-8787.2016050006683

4. Bauman AE, Reis RS, Sallis JF, Wells JC, Loos RJF, Martin BW. Correlates of physical activity: why are some people physically active and others not? Lancet. 2012;380(9838):258-71. https://doi.org/10.1016/S0140-6736(12)60735-1

5. Pereira R, Santos R, Póvoas S, Silva P. Environment perception and leisure-time physical activity in Portuguese high school students. Prev Med Rep. 2018;10:221-6. https://doi.org/10.1016/j.pmedr.2017.10.013

6. Oyeyemi AL, Ishaku CM, Deforche B, Oyeyemi AY, De Bourdeaudhuij I, Van Dyck D. Perception of built environmental factors and physical activity among adolescents in Nigeria. Int J Behav Nutr Phys Act. 2014;11(1):56. https://doi.org/10.1186/1479-5868-11-56 
7. Hino AAF, Reis RS, Sarmiento OL, Parra DC, Brownson RC. The built environment and recreational physical activity among adults in Curitiba, Brazil. Prev Med. 2011;52(6):419-22. https://doi.org/10.1016/j.ypmed.2011.03.019

8. Rech CR, Reis RS, Hino AAF, Hallal PC. Personal, social and environmental correlates of physical activity in adults from Curitiba, Brazil. Prev Med. 2014;58:53-7. https://doi.org/10.1016/j.ypmed.2013.10.023

9. Florindo AA, Barrozo LV, Cabral-Miranda W, Rodrigues EQ, Turrell G, Goldbaum M, et al. Public open spaces and leisure-time walking in Brazilian adults. Int J Environ Res Public Health. 2017;14(6):553. https://doi.org/10.3390/ijerph14060553

10. Hallal PC, Reis RS, Parra DC, Hoehner C, Brownson RC, Simoes EJ. Association between perceived environmental attributes and physical activity among adults in Recife, Brazil. J Phys Act Health. 2010;7 Suppl 2:S213-22. https://doi.org/10.1123/jpah.7.s2.s213

11. Mendonça G, Florindo AA, Rech CR, Freitas DKS, Cazuza J, Farias Júnior JC. Perceived neighborhood environmental characteristics and different types of physical activity among Brazilian adolescents. J Sports Sci. 2017;36(9):1068-75. https://doi.org/10.1080/02640414.2017.1356024

12. Silva ICM, Hino AA, Lopes A, Ekelund U, Brage S, Gonçalves $\mathrm{H}$, et al. Built environment and physical activity: domain-and activity-specific associations among Brazilian adolescents. BMC Public Health. 2017;17(1):616. https://doi.org/10.1186/s12889-017-4538-7

13. Lima AV, Fermino RC, Oliveira MP, Rodriguez Anez CR, Reis RS. [Perceived distance to recreational facilities and the association with physical activity and exercise among adolescents in Curitiba, Parana State, Brazil]. Cad Saude Publica. 2013;29(8):1507-21. Portuguese. https://doi.org/10.1590/0102-311X00175912

14. Instituto Brasileiro de Geografia e Estatística. Cidades: Porto Alegre. Rio de Janeiro: IBGE; 2018 [cited 2018 Nov 20]. Available from: https://cidades.ibge.gov.br/brasil/rs/portoalegre/panorama

15. Instituto Nacional de Estudos e Pesquisas Educacionais Anísio Teixeira. Censo escolar, Rio Grande do Sul, Porto Alegre. Brasília, DF: INEP; 2014 [cited 2018 Nov 20]. Available from: http://portal.inep.gov.br/basica-censo-escolarmatricula

16. Gaya A. Ciências do movimento humano: introdução à metodologia da pesquisa. Porto Alegre: Artmed; 2008.

17. Sawyer SM, Azzopardi PS, Wickremarathne D, Patton GC. The age of adolescence. Lancet Child Adolesc Health. 2018;2(3):223-8. https://doi.org/10.1016/S2352-4642(18)30022-1

18. Craig CL, Marshall AL, Sjöström M, Bauman AE, Booth ML, Ainsworth BE, et al. International physical activity questionnaire: 12-Country reliability and validity. Med Sci Sports Exerc. 2003;35(8):1381-95. https://doi.org/10.1249/01.MSS.0000078924.61453.FB

19. Hallal PC, Gomez LF, Parra DC, Lobelo F, Mosquera J, Florindo AA, et al. Lessons learned after 10 years of IPAQ use in Brazil and Colombia. J Phys Act Health. 2010;7 Suppl 2:S259-64. https://doi.org/10.1123/jpah.7.s2.s259

20. Rosenberg D, Ding D, Sallis JF, Kerr J, Norman GJ, Durant N, et al. Neighborhood Environment Walkability Scale for Youth (NEWS-Y): reliability and relationship with physical activity. Prev Med. 2009;49(2-3):213-8. https://doi.org/10.1016/j.ypmed.2009.07.011

21. Lima AV, Rech CR, Reis RS. [Semantic, item, and conceptual equivalence of the Brazilian version of the Neighborhood Environment Walkability Scale for Youth (NEWS-Y)]. Cad Saude Publica. 2013;29(12):2547-53. https://doi.org/10.1590/0102-311x00182512

22. Sallis JF. Scoring for the Neighborhood Environment Walkability Scale - Youth (NEWS-Y). San Diego, Cal: UC San Diego, School of Medicine; 2009 [cited 2018 Ago 5]. Available from: http://sallis.ucsd.edu/Documents/Measures_documents/NEWS_Y_scoring.pdf

23. Associação Brasileira de Empresa de Pesquisa. Critério de classificação econômica. São Paulo: ABEP; 2015 [cited 2018 Ago 5]. Available from: http://www.abep.org/criterio-brasil

24. Fueyo JL, Garcia LMT, Mamondi V, Alencar GP, Florindo AA, Berra S. Neighborhood and family perceived environments associated with children's physical activity and body mass index. Prev Med. 2016;82:35-41. https://doi.org/10.1016/j.ypmed.2015.11.005

25. De Meester F, Van Dyck D, De Bourdeaudhuij I, Deforche B, Sallis JF, Cardon G. Active living neighborhoods: is neighborhood walkability a key element for Belgian adolescents? BMC Public Health. 2012;12:7. https://doi.org/10.1186/1471-2458-12-7 
26. Sallis JF, Conway TL, Cain KL, Carlson JA, Frank LD, Kerr J, et al. Neighborhood built environment and socioeconomic status in relation to physical activity, sedentary behavior, and weight status of adolescents. Prev Med. 2018;110:47-54. https://doi.org/10.1016/j.ypmed.2018.02.009

27. Rees-Punia E, Hathaway ED, Gay JL. Crime, perceived safety, and physical activity: a meta-analysis. Prev Med. 2017;111:307-13. https://doi.org/10.1016/j.ypmed.2017.11.017

28. Mitáš J, Sas-Nowosielski K, Groffik D, Frömel K. The safety of the neighborhood environment and physical activity in Czech and Polish adolescents. Int J Environ Res Public Health. 2018;15(1):126. https://doi.org/10.3390/ijerph15010126

29. Kowaleski-Jones L, Fan JX, Wen M, Hanson H. Neighborhood context and youth physical activity: differential associations by gender and age. Am J Health Promot. 2017;31(5):426-34. https://doi.org/10.1177/0890117116667353

30. Lee LL, Kuo YL, Chan ESY. The association between built environment attributes and physical activity in east Asian adolescents: a systematic review. Asia Pac J Public Health. 2016;28(3):206-18. https://doi.org/10.1177/1010539516628174

Funding: This work was supported by National Counsel of Technological and Scientific Development (CNPQ), Brazil (n³05200/2013-5). Scholarship from the Brazilian government by from Coordination for the Improvement of Higher Education Personnel (CAPES). JM-was supported by grants: FCT: SFRH/BSAB/142983/2018 and UID/DTP/00617/2019 as well as Scholarship Program of Santander Universities (2018).

Authors' Contributions: Study design and conception: AFD, ARG, MPS, CB, JM, ACAG. Data collection, analysis and interpretation: AFD, CB, ANP, CFF, TMM. Preparation or revision of the manuscript: AFD, CB, CFF, JM, MPS, ACAG. All authors have approved the final version of the study.

Conflict of interests: The authors declare no conflict of interest. 